\title{
Lévy Flights over Quantum Paths
}

\author{
Nick Laskin* \\ New York University \\ Courant Institute of Mathematical Sciences \\ 251 Mercer Street, NY 10012
}

\begin{abstract}
An impact of integration over the paths of the Lévy flights on the quantum mechanical kernel has been studied. Analytical expression for a free particle kernel has been obtained in terms of the Fox $\mathrm{H}$ function. A new equation for the kernel of a partical in the box has been found. New general results include the well known quantum formulae for a free particle kernel and particle in box kernel.
\end{abstract}

PACS number(s): 03.65.-w, 03.65. Db, 05.30.-d, 05.40. Fb

Keywords: Lévy flights, path integral, quantum mechanical kernel.

\section{Introduction}

In past decade it has been realized that the understanding of complex quantum and classical physic phenomena has required the implementation of the Lévy flights random process [1, 2]. It is well known that a position of diffusive particle increases as square root in time, $x(t) \sim t^{1 / 2}$. The square root law is attribute of the Brownian motion model for diffusion. However for complex quantum and classical physic phenomena this temporal diffusive behavior has not been observed. Instead, more general evolution law $x(t) \sim t^{1 / \alpha}$ with $0<\alpha \leq 2$ has been found. Thus, the well known diffusion law $x(t) \sim t^{1 / 2}$ is included as a special case at $\alpha=2$. The mathematical model to describe $1 / \alpha$ diffusion scaling is known as Lévy flights. The Lévy

*E-mail: laskin@cims.nyu.edu 
flights is general framework to study 'unusual diffusion' in terms of fractional Fokker-Planck equation [1], 2].

The scaling $1 / \alpha$ law assigned to the Lévy flights has been empirically observed in laser cooling of atoms [3], in ion dynamics in optical lattice 4], in anomalous transport [5], in the measurement of the momentum of cold cesium atoms in a periodically pulsed standing wave of light [6]. The Lévy flights are widely used to model a variety of physical phenomena such as kinetics and transport in classical systems, anomalous diffusion, chaotic dynamics, plasma physics, dynamics of economic indexes, biology and physiology, social science (see for example, 1], 2] and references there).

So too, the Feynman path integral ought to be generalized to describe complex dynamic phenomena at the quantum level. The Feynman path integral is in fact the integration over Brownian-like quantum mechanical paths [7, [8]. To generalize the Feynman path integral approach the integration has been expanded from Brownian-like to Lévy flights paths [9], 10].

In this paper we study an impact of integration over the paths of the Lévy flights on the structure of a free particle quantum mechanical kernel. The kernel has been expressed in terms of Fox's $H$-function.

The paper is organized as follows. We introduce path integral over the Lévy paths in Sec.2. It has been shown in Sec.2 that the Levy path integral goes over into the Feynman path integral when self-similarity (or the Lévy) index $\alpha=2$. Thus, our new results go over into the well known quantum equations at the special case $\alpha=2$.

Sec.3 deals with a free particle quantum kernel. We have found Fox's $H$-function representation for a free particle quantum kernel. The representation gives us an option to use many well established equations, identities, integrals involving the $H$-function for practical Lévy path integral calculations.

Sec.4 summarizes the Laplace and the energy-time transforms and the momentum representation of the Lévy quantum mechanical kernel.

New equation for the quantum kernel of a particle in a box has been found and discussed in Sec.5. 


\section{Path Integral}

\subsection{Feynman path integral}

If a particle at an initial time $t_{a}$ starts from the point $x_{a}$ and goes to a final point $x_{b}$ at time $t_{b}$, we will say simply that the particle goes from $a$ to $b$ and its trajectory (path) $x(t)$ will have the property that $x\left(t_{a}\right)=x_{a}$ and $x\left(t_{b}\right)=x_{b}$. In quantum mechanics, then, we will have an quantum-mechanical kernel, which we may write as $K\left(x_{b} t_{b} \mid x_{a} t_{a}\right)$, to get from the point $a$ to the point $b$. This will be the sum over all of the trajectories that go between that end points and of a contribution from each. Following Feynman [7] we write the kernel in the form

$$
K\left(x_{b} t_{b} \mid x_{a} t_{a}\right)=\int_{x\left(t_{a}\right)=x_{a}}^{x\left(t_{b}\right)=x_{b}} D x(\tau) \exp \left\{\frac{i}{\hbar} S(x)\right\}
$$

where $S(x)$ is classical mechanical action considered as the functional of a particle trajectory $x(\tau)$

$$
\left.S(\tau)=\int_{t_{a}}^{t_{b}} d \tau\left(\frac{m \dot{x}^{2}}{2}-V(x(\tau), \tau)\right)\right)
$$

here $V(x, t)$ is the potential energy and $\int_{x\left(t_{a}\right)=x_{a}}^{x\left(t_{b}\right)=x_{b}} D x(\tau) \ldots$ is defined as follows

$$
\int_{x\left(t_{a}\right)=x_{a}}^{x\left(t_{b}\right)=x_{b}} D x(\tau) \ldots=\lim _{N \rightarrow \infty}\left(\frac{2 \pi i \hbar \varepsilon}{m}\right)^{-1 / 2} \int_{-\infty}^{\infty} \prod_{j=1}^{N-1}\left(\frac{2 \pi i \hbar \varepsilon}{m}\right)^{-1 / 2} d x_{j} \ldots, \quad \varepsilon=\frac{t_{b}-t_{a}}{N} .
$$

Feynman's original path integral approach to non-relativistic quantum mechanics is based on the fundamental equations (11)-(3).

Then the equation

$$
\psi\left(x_{b}, t_{b}\right)=\int_{-\infty}^{\infty} d x_{a} K\left(x_{b} t_{b} \mid x_{a} t_{a}\right) \psi\left(x_{a}, t_{a}\right)
$$


gives the wave function $\psi\left(x_{b}, t_{b}\right)$ at a time $t_{b}$ in terms of the wave function $\psi\left(x_{a}, t_{a}\right)$ at a time $t_{a}$. This fundamental equation describes the evolution of the quantum mechanical system in the terms of the wave function.

\subsubsection{The free particle}

To calculate the quantum-mechanical amplitude $K^{(0)}\left(x_{b} t_{b} \mid x_{a} t_{a}\right)$ for a free particle, $V(x)=0$ we will follow Feynman [7] ,

$$
K^{(0)}\left(x_{b} t_{b} \mid x_{a} t_{a}\right)=\int_{x\left(t_{a}\right)=x_{a}}^{x\left(t_{b}\right)=x_{b}} D x(\tau) \exp \left\{\frac{i}{\hbar} \int_{t_{a}}^{t_{b}} d \tau \frac{m \dot{x}^{2}}{2}\right\} .
$$

With the help of Eqs.(11)-(3) we have

$$
\begin{aligned}
K^{(0)}\left(x_{b} t_{b} \mid x_{a} t_{a}\right)= & \lim _{N \rightarrow \infty}\left(\frac{2 \pi i \hbar \varepsilon}{m}\right)^{-1 / 2} \int_{-\infty}^{\infty} \prod_{j=1}^{N-1}\left(\frac{2 \pi i \hbar \varepsilon}{m}\right)^{-1 / 2} d x_{j} \times \\
& \prod_{j=1}^{N} \exp \left\{\frac{i m}{2 \hbar \varepsilon}\left(x_{j}-x_{j-1}\right)^{2}\right\} .
\end{aligned}
$$

The calculation is carried out as follows. It is easy to see that ${ }^{1}$

$$
\begin{gathered}
\left(\frac{2 \pi i \hbar \varepsilon}{m}\right)^{-1 / 2} \int_{-\infty}^{\infty} d x_{1} \exp \left\{\frac{i m}{2 \hbar \varepsilon}\left[\left(x_{2}-x_{1}\right)^{2}+\left(x_{1}-x_{a}\right)^{2}\right]\right\}= \\
\left(\frac{2 \pi i \hbar 2 \varepsilon}{m}\right)^{-1 / 2} \exp \left\{\frac{i m}{2 \hbar 2 \varepsilon}\left(x_{2}-x_{a}\right)^{2}\right\} .
\end{gathered}
$$

Next we multiply this result by

$$
\left(\frac{2 \pi i \hbar \varepsilon}{m}\right)^{-1 / 2} \exp \left\{\frac{i m}{2 \hbar \varepsilon}\left(x_{3}-x_{2}\right)^{2}\right\}
$$

${ }^{1}$ Here we use the definite integral

$$
\int_{-\infty}^{\infty} d x e^{-a x^{2}+b x}=\sqrt{\frac{\pi}{a}} e^{b^{2} / 4 a}
$$


and integrate again, this time over $x_{2}$. The result of integration is similar to that of Eq.(7), except that $\left(x_{2}-x_{a}\right)^{2}$ becomes $\left(x_{3}-x_{a}\right)^{2}$ and the expression $2 \varepsilon$ is replaced by $3 \varepsilon$ in two places. Thus, we get

$$
\left(\frac{2 \pi i \hbar 3 \varepsilon}{m}\right)^{-1 / 2} \exp \left\{\frac{i m}{2 \hbar 3 \varepsilon}\left(x_{3}-x_{a}\right)^{2}\right\} \text {. }
$$

In this way a recursion procedure is established which after $N-1$ steps gives

$$
\left(\frac{2 \pi i \hbar N \varepsilon}{m}\right)^{-1 / 2} \exp \left\{\frac{i m}{2 \hbar N \varepsilon}\left(x_{a}-x_{0}\right)^{2}\right\} .
$$

Since $N \varepsilon=t_{b}-t_{a}$, it is easy to see that the final result after $N-1$ steps is

$$
K^{(0)}\left(x_{b} t_{b} \mid x_{a} t_{a}\right)=\left(\frac{2 \pi i \hbar\left(t_{b}-t_{a}\right)}{m}\right)^{-1 / 2} \cdot \exp \left\{\frac{i m\left(x_{b}-x_{a}\right)^{2}}{2 \hbar\left(t_{b}-t_{a}\right)}\right\} .
$$

The Eq.(8) implies

$$
\Delta x \propto\left(\frac{\hbar}{m}\right)^{1 / 2}(\Delta t)^{1 / 2}
$$

which means that space displacement $\Delta x=x_{b}-x_{a}$ and the time $\Delta t=$ $t_{b}-t_{a}$ scale are governed by the Brownian motion law.

In terms of the Fourier integral the Feynman kernel $K^{(0)}\left(x_{b} t_{b} \mid x_{a} t_{a}\right)$ given by Eq.(8) can be written as

$$
K^{(0)}\left(x_{b} t_{b} \mid x_{a} t_{a}\right)=\frac{1}{2 \pi \hbar} \int_{-\infty}^{\infty} d p \cdot \exp \left\{i \frac{p\left(x_{b}-x_{a}\right)}{\hbar}-i \frac{p^{2}\left(t_{b}-t_{a}\right)}{2 m \hbar}\right\} .
$$

\subsection{The path integral over the Lévy paths}

The "natural" way to generalize Eq.(9]) is [9], [10]

$$
K_{L}^{(0)}\left(x_{b} t_{b} \mid x_{a} t_{a}\right)=\frac{1}{2 \pi \hbar} \int_{-\infty}^{\infty} d p \cdot \exp \left\{i \frac{p\left(x_{b}-x_{a}\right)}{\hbar}-i \frac{D_{\alpha}|p|^{\alpha}\left(t_{b}-t_{a}\right)}{\hbar}\right\}
$$


where $D_{\alpha}$ is "fractional quantum diffusion coefficient" physical dimension of which is

$$
\left[D_{\alpha}\right]=\operatorname{erg}^{1-\alpha} \cdot \mathrm{cm}^{\alpha} \cdot \sec ^{-\alpha}
$$

and $\alpha$ is the Levy index, and we consider $1<\alpha \leq 2$.

It is easy to see from Eq.(10) that the scaling relation between space displacement $\Delta x=x_{b}-x_{a}$ and the time increment $\Delta t=t_{b}-t_{a}$ has a form

$$
\Delta x \propto\left(\hbar^{\alpha-1} D_{\alpha}\right)^{1 / \alpha}(\Delta t)^{1 / \alpha} .
$$

This scaling relation implies that space displacement and the time increment are governed by the Lévy flights law.

Using Eq.(10) we can define the path integral in the phase space representation by the following way

$$
\begin{gathered}
\int_{x\left(t_{a}\right)=x_{a}}^{x\left(t_{b}\right)=x_{b}} \mathrm{D} x(\tau) \int \mathrm{D} p(\tau) \ldots= \\
=\lim _{N \rightarrow \infty} \int_{-\infty}^{\infty} d x_{1} \ldots d x_{N-1} \frac{1}{(2 \pi \hbar)^{N}} \int_{-\infty}^{\infty} d p_{1} \ldots d p_{N} \times \\
\exp \left\{i \frac{p_{1}\left(x_{1}-x_{a}\right)}{\hbar}-i \frac{D_{\alpha} \varepsilon\left|p_{1}\right|^{\alpha}}{\hbar}\right\} \times \ldots \times \exp \left\{i \frac{p_{N}\left(x_{b}-x_{N-1}\right)}{\hbar}-i \frac{D_{\alpha} \varepsilon\left|p_{N}\right|^{\alpha}}{\hbar}\right\} \ldots
\end{gathered}
$$

here again $\varepsilon=\left(t_{b}-t_{a}\right) / N$. Then the kernel $K_{L}\left(x_{b} t_{b} \mid x_{a} t_{a}\right)$ defined by Eq.(10) can be written as

$$
\begin{gathered}
K_{L}\left(x_{b} t_{b} \mid x_{a} t_{a}\right)=\lim _{N \rightarrow \infty} \int_{-\infty}^{\infty} d x_{1} \ldots d x_{N-1} \frac{1}{(2 \pi \hbar)^{N}} \int_{-\infty}^{\infty} d p_{1} \ldots d p_{N} \times \\
\exp \left\{\frac{i}{\hbar} \sum_{j=1}^{N} p_{j}\left(x_{j}-x_{j-1}\right)\right\} \times \exp \left\{-\frac{i}{\hbar} D_{\alpha} \varepsilon \sum_{j=1}^{N}\left|p_{j}\right|^{\alpha}-\frac{i}{\hbar} \varepsilon \sum_{j=1}^{N} V\left(x_{j}, j \varepsilon\right)\right\} .
\end{gathered}
$$

In the continuum limit $N \rightarrow \infty, \quad \varepsilon \rightarrow 0$ we have 


$$
\begin{gathered}
K_{L}\left(x_{b} t_{b} \mid x_{a} t_{a}\right)= \\
\int_{x\left(t_{a}\right)=x_{a}}^{x\left(t_{b}\right)=x_{b}} \mathrm{D} x(\tau) \int \mathrm{D} p(\tau) \exp \left\{\frac{i}{\hbar} \int_{t_{a}}^{t_{b}} d \tau\left[p(\tau) \dot{x}(\tau)-H_{\alpha}(p(\tau), x(\tau), \tau]\right\},\right.
\end{gathered}
$$

where the phase space path integral $\int_{x\left(t_{a}\right)=x_{a}}^{x\left(t_{b}\right)=x_{b}} \mathrm{D} x(\tau) \int \mathrm{D} p(\tau) \ldots$ is given by Eq.(11), $\dot{x}$ denotes the time derivative, $H_{\alpha}$ is the Hamiltonian

$$
H_{\alpha}(p, x)=D_{\alpha}|p|^{\alpha}+V(x, t)
$$

with the replacement $p \rightarrow p(\tau), x \rightarrow x(\tau)$ and $\{p(\tau), x(\tau)\}$ is the particle trajectory in phase space. The hermiticity property of the Hamiltonian $H_{\alpha}$ has been discussed in [11.

The exponential in Eq.(12) can be written as $\exp \left\{\frac{i}{\hbar} S_{\alpha}(p, x)\right\}$ if we introduce canonical action $S_{\alpha}(p, x)$ for the trajectory $p(t), x(t)$ in phase space

$$
S_{\alpha}(p, x)=\int_{t_{a}}^{t_{b}} d \tau\left(p(\tau) \dot{x}(\tau)-H_{\alpha}(p(\tau), x(\tau), \tau)\right) .
$$

Since the coordinates $x_{0}, x_{N}$ in the definition (111) are fixed at their initial and final points, $x_{0}=x_{a}$ and $x_{N}=x_{b}$, the all possible trajectories in Eqs.(11), (12) satisfy the boundary condition $x\left(t_{b}\right)=x_{b}, x\left(t_{a}\right)=x_{a}$. We see that the definition given by Eq.(11) includes one more $p_{j}$-integrals than $x_{j}$-integrals. Indeed, while $x_{0}$ and $x_{N}$ are held fixed and the $x_{j}$-integrals are done for $j=1, \ldots, N-1$, each increment $x_{j}-x_{j-1}$ is accompanied by one $p_{j}$-integral for $j=1, \ldots, N$. The above observed asymmetry is a consequence of the particular boundary condition. Namely, the end points are fixed in the position (coordinate) space. There exists the possibility of proceeding in a conjugate way keeping the initial $p_{a}$ and final $p_{b}$ momenta fixed. The associated kernel can be derived going through the same steps as before but working in the momentum representation (see, for example, [12]).

Taking into account Eq.(10) it is easily to check on directly the consistency condition

$$
K_{L}^{(0)}\left(x_{b} t_{b} \mid x_{a} t_{a}\right)=\int_{-\infty}^{\infty} d x^{\prime} K_{L}^{(0)}\left(x_{b} t_{b} \mid x^{\prime} t^{\prime}\right) \cdot K_{L}^{(0)}\left(x^{\prime} t^{\prime} \mid x_{a} t_{a}\right)
$$


This is a special case of the general quantum-mechanical rule: the kernels for events occurring in succession in time satisfy

$$
K_{L}\left(x_{b} t_{b} \mid x_{a} t_{a}\right)=\int_{-\infty}^{\infty} d x^{\prime} K_{L}\left(x_{b} t_{b} \mid x^{\prime} t^{\prime}\right) \cdot K_{L}\left(x^{\prime} t^{\prime} \mid x_{a} t_{a}\right)
$$

where $K_{L}\left(x_{b} t_{b} \mid x^{\prime} t^{\prime}\right)$ is defined by Eq.(12).

\section{Fox $H$ function representation for a free particle kernel $K_{L}^{(0)}\left(x_{b} t_{b} \mid x_{a} t_{a}\right)$}

Let us show how a free particle quantum mechanical kernel $K_{L}^{(0)}\left(x_{b} t_{b} \mid x_{a} t_{a}\right)$ defined by Eq.(10) can be expressed in the terms of the Fox's $H$-function [13], [14], 15]. Apart from the quiet natural way in which the Fox's $H$-function enters in the path integral technique over the Lévy flights, its derivatives and integrals are easily calculated by formally manipulating the parameters in the $H$-function. That is why the $H$-Fox's function is so important and useful for the path integration over the Lévy paths. Note that $H$-function bears the name of their discoverer Fox [13] although it has been known at least since 1888 (see [14]).

To present a free particle kernel $K_{L}^{(0)}\left(x_{b} t_{b} \mid x_{a} t_{a}\right)$ in terms of the Fox $H$ function we accept the following strategy. Starting from the representation (10) we obtain the Mellin transform of the quantum mechanical kernel. Comparing of the inverse Mellin transform with the definition of the Fox's function [13], 14 yields the desired expression in terms of "known" function, i.e. Fox's $H$-function.

Introducing for simplicity the notations

$$
x \equiv x_{b}-x_{a}, \quad \tau \equiv t_{b}-t_{a},
$$

we rewrite Eq.(10)

$$
K_{L}^{(0)}(x, \tau)=\frac{1}{2 \pi \hbar} \int_{-\infty}^{\infty} d p \cdot \exp \left\{i \frac{p x}{\hbar}-i \frac{D_{\alpha}|p|^{\alpha} \tau}{\hbar}\right\} .
$$

One can see that the relation

$$
K_{L}^{(0)}(x, \tau)=K_{L}^{(0)}(-x, \tau),
$$


holds. Hence, it is sufficient to consider $K_{L}^{(0)}(x, \tau)$ for $x \geq 0$ only. Further, we will use the following definitions of the Mellin

$$
\hat{K}(s, \tau)=\int_{0}^{\infty} d x x^{s-1} K_{L}(x, \tau),
$$

and the inverse Mellin transforms

$$
K_{L}(x, \tau)=\frac{1}{2 \pi i} \int_{c-i \infty}^{c+i \infty} d s x^{-s} \stackrel{\wedge}{K}(s, \tau),
$$

where the integration path is the straight line from $c-i \infty$ to $c+i \infty$ with $0<c<1$.

The Mellin transform of the $K_{L}^{(0)}(x, \tau)$ is

$$
\begin{gathered}
\hat{K_{L}^{(0)}}(s, \tau)=\int_{0}^{\infty} d x x^{s-1} K_{L}^{(0)}(x, \tau)= \\
\frac{1}{2 \pi \hbar} \int_{0}^{\infty} d x x^{s-1} \int_{-\infty}^{\infty} d p \cdot \exp \left\{i \frac{p x}{\hbar}-i \frac{D_{\alpha}|p|^{\alpha} \tau}{\hbar}\right\} .
\end{gathered}
$$

By a change of variables

$$
p \rightarrow\left(\frac{\hbar}{i D_{\alpha} \tau}\right)^{1 / \alpha} \varsigma, \quad x \rightarrow \hbar\left(\frac{\hbar}{i D_{\alpha} \tau}\right)^{-1 / \alpha} \xi
$$

$\hat{K^{(0)}}(s, \tau)$ goes over to

$$
\hat{K_{L}^{(0)}}(s, \tau)=\frac{1}{2 \pi}\left(\frac{\hbar}{\left(\hbar / i D_{\alpha} \tau\right)^{1 / \alpha}}\right)^{s-1} \int_{0}^{\infty} d \xi \xi^{s-1} \int_{-\infty}^{\infty} d \varsigma \exp \left\{i \varsigma \xi-|\varsigma|^{\alpha}\right\} .
$$

The integrals over $d \xi$ and $d \varsigma$ can be evaluated by using the equations (3.3) and (3.6) of the Ref. [16]. Indeed, we have

$$
\int_{0}^{\infty} d \xi \xi^{s-1} \int_{0}^{\infty} d \varsigma \exp \left\{i \varsigma \xi-\varsigma^{\alpha}\right\}=\frac{4}{s-1} \sin \frac{\pi(s-1)}{2} \Gamma(s) \Gamma\left(1-\frac{s-1}{\alpha}\right)
$$


where $s-1<\alpha \leq 2$ and $\Gamma(s)$ is the gamma function ${ }^{2}$.

Inserting Eq.(21) into Eq.(20) and using the functional relations for the gamma function, $\Gamma(1-z)=-z \Gamma(-z)$ and $\Gamma(z) \Gamma(1-z)=\pi / \sin \pi z$, we find

$$
\hat{K_{L}^{(0)}}(s, \tau)=\frac{1}{\alpha}\left(\frac{\hbar}{\left(\hbar / i D_{\alpha} \tau\right)^{1 / \alpha}}\right)^{s-1} \frac{\Gamma(s) \Gamma\left(\frac{1-s}{\alpha}\right)}{\Gamma\left(\frac{1-s}{2}\right) \Gamma\left(\frac{1+s}{2}\right)} .
$$

Then the inverse Mellin transform gives a free particle quantum mechanical kernel $K_{L}^{(0)}(x, \tau)$

$$
\begin{gathered}
K_{L}^{(0)}(x, \tau)=\frac{1}{2 \pi i} \int_{c-i \infty}^{c+i \infty} d s x^{-s} \hat{K}_{L}^{(0)}(s, \tau)= \\
\frac{1}{2 \pi i} \frac{1}{\alpha} \cdot \int_{c-i \infty}^{c+i \infty} d s\left(\frac{\hbar}{\left(\hbar / i D_{\alpha} \tau\right)^{1 / \alpha}}\right)^{s-1} x^{-s} \frac{\Gamma(s) \Gamma\left(\frac{1-s}{\alpha}\right)}{\Gamma\left(\frac{1-s}{2}\right) \Gamma\left(\frac{1+s}{2}\right)},
\end{gathered}
$$

where the integration path is the straight line from $c-i \infty$ to $c+i \infty$ with $0<c<1$. Replacing $s$ by $-s$ we find

$$
\begin{gathered}
K_{L}^{(0)}(x, \tau)= \\
\frac{1}{\alpha}\left(\frac{\hbar}{\left(\hbar / i D_{\alpha} \tau\right)^{1 / \alpha}}\right)^{-1} \frac{1}{2 \pi i} \int_{-c-i \infty}^{-c+i \infty} d s\left(\frac{1}{\hbar}\left(\frac{\hbar}{i D_{\alpha} \tau}\right)^{1 / \alpha} x\right)^{s} \frac{\Gamma(-s) \Gamma\left(\frac{1+s}{\alpha}\right)}{\Gamma\left(\frac{1+s}{2}\right) \Gamma\left(\frac{1-s}{2}\right)} .
\end{gathered}
$$

The path of integration may be deformed into one running clockwise around $R_{+}-c$. Comparison with the definition of the Fox's $H$-function (see Eqs.(58)-(60), Appendix A) yields

$$
\begin{gathered}
K_{L}^{(0)}(x, \tau)= \\
\frac{1}{\alpha}\left(\frac{\hbar}{\left(\hbar / i D_{\alpha} \tau\right)^{1 / \alpha}}\right)^{-1} H_{2,2}^{1,1}\left[\frac{1}{\hbar}\left(\frac{\hbar}{i D_{\alpha} \tau}\right)^{1 / \alpha} x \mid \begin{array}{c}
(1-1 / \alpha, 1 / \alpha),(1 / 2,1 / 2) \\
(0,1),(1 / 2,1 / 2)
\end{array}\right] .
\end{gathered}
$$

\footnotetext{
${ }^{2}$ The gamma function $\Gamma(s)$ has the familiar integral representation $\Gamma(s)=\int_{0}^{\infty} d t t^{s-1} e^{-t}$,
} Res $>0$. 
Because of the Property 12.2.5 (see Appendix A) of the Fox's $H$-function the kernel $K_{L}^{(0)}(x, \tau)$ becomes

$$
K_{L}^{(0)}(x, \tau)=\frac{1}{\alpha x} H_{2,2}^{1,1}\left[\frac{1}{\hbar}\left(\frac{\hbar}{i D_{\alpha} \tau}\right)^{1 / \alpha} x \mid \begin{array}{c}
(1,1 / \alpha),(1,1 / 2) \\
(1,1),(1,1 / 2)
\end{array}\right], \quad x>0
$$

Hence, for any $x$ we have,

$$
K_{L}^{(0)}(x, \tau)=\frac{1}{\alpha x} H_{2,2}^{1,1}\left[\frac{1}{\hbar}\left(\frac{\hbar}{i D_{\alpha} \tau}\right)^{1 / \alpha}|x| \mid \begin{array}{c}
(1,1 / \alpha),(1,1 / 2) \\
(1,1),(1,1 / 2)
\end{array}\right]
$$

Remembering Eqs.(16), (17) finally yields

$$
\begin{gathered}
K_{L}^{(0)}\left(x_{b} t_{b} \mid x_{a} t_{a}\right)= \\
\frac{1}{\alpha\left|x_{b}-x_{a}\right|} H_{2,2}^{1,1}\left[\frac{1}{\hbar}\left(\frac{\hbar}{i D_{\alpha}\left(t_{b}-t_{a}\right)}\right)^{1 / \alpha}\left|x_{b}-x_{a}\right| \mid \begin{array}{c}
(1,1 / \alpha),(1,1 / 2) \\
(1,1),(1,1 / 2)
\end{array}\right] .
\end{gathered}
$$

This is new equation for 1-D free particle quantum kernel $K_{L}^{(0)}\left(x_{b} t_{b} \mid x_{a} t_{a}\right)$. Let us show that Eq.(26) includes as a particular case at $\alpha=2$ the well known Feynman quantum mechanical kernel given by Eq.(8). Indeed, setting in Eq.(26) $\alpha=2$ and applying the series expansion Eq.(63) for the function $H_{2,2}^{1,1}\left[\frac{1}{\hbar}\left(\frac{\hbar}{D_{2} \tau}\right)^{1 / 2}|x| \mid \underset{(1,1),(1,1 / 2)}{(1,1 / 2),(1,1 / 2)}\right]$ we have

$$
\left.K_{L}^{(0)}(x, \tau)\right|_{\alpha=2}=\frac{1}{2 \hbar}\left(\frac{\hbar}{i D_{2} \tau}\right)^{1 / 2} \sum_{k=0}^{\infty}\left(-\frac{1}{\hbar}\left(\frac{\hbar}{i D_{2} \tau}\right)^{1 / 2}\right)^{k} \frac{|x|^{k}}{k !} \frac{1}{\Gamma\left(\frac{1-k}{2}\right)} .
$$

By substituting $k \rightarrow 2 l$ we obtain

$$
\left.K_{L}^{(0)}(x, \tau)\right|_{\alpha=2}=\frac{1}{2 \hbar}\left(\frac{\hbar}{i D_{2} \tau}\right)^{1 / 2} \sum_{l=0}^{\infty}\left(-\frac{1}{\hbar}\left(\frac{\hbar}{i D_{2} \tau}\right)^{1 / 2}\right)^{2 l} \frac{x^{2 l}}{(2 l) !} \frac{1}{\Gamma\left(\frac{1}{2}-l\right)}
$$

Taking into account the identity 


$$
\Gamma\left(\frac{1}{2}+z\right) \Gamma\left(\frac{1}{2}-z\right)=\frac{\pi}{\cos \pi z},
$$

and applying the Gauss multiplication formula

$$
\Gamma(2 l)=\sqrt{\frac{2^{4 l-1}}{2 \pi}} \Gamma(l) \Gamma\left(l+\frac{1}{2}\right),
$$

we find that

$$
(2 l) ! \Gamma\left(\frac{1}{2}-l\right)=\frac{\sqrt{\pi}}{(-1)^{l}}(2)^{2 l} l ! .
$$

With the help of Eq.(28) the kernel $\left.K_{L}^{(0)}(x, \tau)\right|_{\alpha=2}$ can be rewritten as

$$
\begin{aligned}
\left.K_{L}^{(0)}(x, \tau)\right|_{\alpha=2}= & \frac{1}{2 \sqrt{\pi} \hbar}\left(\frac{\hbar}{i D_{2} \tau}\right)^{1 / 2} \sum_{l=0}^{\infty}\left(-\frac{1}{\hbar}\left(\frac{\hbar}{i D_{2} \tau}\right)^{1 / 2}\right)^{2 l} \frac{(-1)^{l} x^{2 l}}{2^{2 l} l !}= \\
& \frac{1}{2 \sqrt{\pi} \hbar}\left(\frac{\hbar}{i D_{2} \tau}\right)^{1 / 2} \exp \left\{-\frac{1}{4} \frac{x^{2}}{\hbar i D_{2} \tau}\right\} .
\end{aligned}
$$

Since $D_{2}=1 / 2 m$ [9], [10] we finally obtain for the Feynman kernel $K^{(0)}(x, \tau)$

$$
\left.K^{(0)}(x, \tau) \equiv K_{L}^{(0)}(x, \tau)\right|_{\alpha=2}=\sqrt{\frac{m}{2 \pi i \hbar \tau}} \exp \left\{\frac{i m x^{2}}{2 \hbar \tau}\right\} .
$$

It turns into Eq.(3-3) of Ref. [7] if we use Eq.(16).

Thus, it is shown that a free particle Feynman kernel can be derived from the general equation (26).

\section{$3.13-\mathrm{D}$ generalization}

The above developments can be generalized to 3-D dimension. It is obviously that a free particle kernel given by Eq.(10) for the 3-D case has the form 


$$
K_{L}^{(0)}\left(\mathbf{r}_{b} t_{b} \mid \mathbf{r}_{a} t_{a}\right)=\frac{1}{(2 \pi \hbar)^{3}} \int d^{3} p \cdot \exp \left\{i \frac{\mathbf{p}\left(\mathbf{r}_{b}-\mathbf{r}_{a}\right)}{\hbar}-i \frac{D_{\alpha}|\mathbf{p}|^{\alpha}\left(t_{b}-t_{a}\right)}{\hbar}\right\}
$$

where $\mathbf{r}$ and $\mathbf{p}$ are the 3 -D vectors.

To express the kernel $K_{L}^{(0)}\left(\mathbf{r}_{b} t_{b} \mid \mathbf{r}_{a} t_{a}\right)$ in the terms of the Fox's $H$-function we write

$$
\begin{gathered}
K_{L}^{(0)}\left(\mathbf{r}_{b} t_{b} \mid \mathbf{r}_{a} t_{a}\right)= \\
\frac{1}{(2 \pi \hbar)^{3}} \int_{0}^{\infty} d p p^{2} \int_{-\pi}^{\pi} d \vartheta \int_{0}^{2 \pi} d \varphi \exp \left\{i \frac{p\left|\mathbf{r}_{b}-\mathbf{r}_{a}\right| \cos \vartheta}{\hbar}-i \frac{D_{\alpha}|\mathbf{p}|^{\alpha}\left(t_{b}-t_{a}\right)}{\hbar}\right\}= \\
\frac{1}{2 \pi^{2} \hbar^{2}\left|\mathbf{r}_{b}-\mathbf{r}_{a}\right|} \int_{0}^{\infty} d p p \sin \left(\frac{p\left|\mathbf{r}_{b}-\mathbf{r}_{a}\right|}{\hbar}\right) \exp \left\{-i \frac{D_{\alpha}|\mathbf{p}|^{\alpha}\left(t_{b}-t_{a}\right)}{\hbar}\right\}
\end{gathered}
$$

With help of the formula

$$
p \sin \left(\frac{p\left|\mathbf{r}_{b}-\mathbf{r}_{a}\right|}{\hbar}\right)=-\hbar \frac{\partial}{\partial\left|\mathbf{r}_{b}-\mathbf{r}_{a}\right|} \cos \left(\frac{p\left|\mathbf{r}_{b}-\mathbf{r}_{a}\right|}{\hbar}\right),
$$

$K_{L}^{(0)}\left(\mathbf{r}_{b} t_{b} \mid \mathbf{r}_{a} t_{a}\right)$ can be written as

$$
K_{L}^{(0)}\left(\mathbf{r}_{b} t_{b} \mid \mathbf{r}_{a} t_{a}\right)=-\left.\frac{1}{2 \pi} \frac{\partial}{\partial x} K_{L}^{(0)}\left(x ; t_{b}-t_{a}\right)\right|_{x=\left|\mathbf{r}_{b}-\mathbf{r}_{a}\right|},
$$

where the kernel $K_{L}^{(0)}\left(x ; t_{b}-t_{a}\right)$ is 1-D kernel given by Eq.(25).

Let us note that Eq.(33) is just a special case of a general relation that holds between the D-mensional and D +2 -mensional Fourier transforms of any isotropic function. An important consequence of Eq.(33) is that it allows us to evaluate the 3D kernel based on Eq.(25) for 1-D quantum kernel. Thus, the problem is to calculate the derivative of the 1-D kernel. Using the Property 12.2.9 (see Appendix A) yields

$$
K_{L}^{(0)}\left(\mathbf{r}_{b} t_{b} \mid \mathbf{r}_{a} t_{a}\right)=
$$




$$
-\frac{1}{2 \pi \alpha} \frac{1}{\left|\mathbf{r}_{b}-\mathbf{r}_{a}\right|^{3}} H_{3,3}^{1,2}\left[\frac{1}{\hbar}\left(\frac{\hbar}{i D_{\alpha}\left(t_{b}-t_{a}\right)}\right)^{1 / \alpha}\left|\mathbf{r}_{b}-\mathbf{r}_{a}\right| \mid \begin{array}{c}
(1,1),(1,1 / \alpha),(1,1 / 2) \\
(1,1),(1,1 / 2),(2,1)
\end{array}\right]
$$

This is new equation for a free particle quantum mechanical 3-D kernel. We see that in comparison with 1-D case the 3-D quantum kernel is expressed in the terms of $H_{3,3}^{1,2}$ Fox's $H$-function.

Let's see that the 3-D kernel given by Eq.(34) goes over into the Feynman 3-D kernel. Setting in Eq.(34) $\alpha=2$ and applying the series expansion Eq.(63) for the function $H_{3,3}^{1,2}$ we have

$$
\begin{gathered}
\left.K_{L}^{(0)}\left(\mathbf{r}_{b} t_{b} \mid \mathbf{r}_{a} t_{a}\right)\right|_{\alpha=2}= \\
-\frac{1}{4 \pi \hbar\left|\mathbf{r}_{b}-\mathbf{r}_{a}\right|} \sum_{k=0}^{\infty} \frac{\Gamma(1+k)}{\Gamma(k) \Gamma\left(\frac{1-k}{2}\right)} \frac{(-1)^{k}}{k !} \cdot\left(\left(\frac{\hbar}{i D_{\alpha}\left(t_{b}-t_{a}\right)}\right)^{1 / 2}\left|\mathbf{r}_{b}-\mathbf{r}_{a}\right|\right)^{1+k} \\
\text { or } \\
\left.K_{L}^{(0)}\left(\mathbf{r}_{b} t_{b} \mid \mathbf{r}_{a} t_{a}\right)\right|_{\alpha=2}= \\
-\frac{1}{4 \pi \hbar} \cdot\left(\frac{\hbar}{i D_{2}\left(t_{b}-t_{a}\right)}\right)^{1 / 2} \frac{\partial}{\partial\left|\mathbf{r}_{b}-\mathbf{r}_{a}\right|} \sum_{k=0}^{\infty} \frac{\left\{-\frac{1}{\hbar}\left(\frac{\hbar}{i D_{2}\left(t_{b}-t_{a}\right)}\right)^{1 / 2}\left|\mathbf{r}_{b}-\mathbf{r}_{a}\right|\right\}^{k}}{k ! \Gamma\left(\frac{1-k}{2}\right)}
\end{gathered}
$$

By substituting $k \rightarrow 2 l$ and using Eq.(28) we observe that there exists the following relation

$$
\sum_{k=0}^{\infty} \frac{\left\{-\frac{1}{\hbar}\left(\frac{\hbar}{i D_{2}\left(t_{b}-t_{a}\right)}\right)^{1 / 2}\left|\mathbf{r}_{b}-\mathbf{r}_{a}\right|\right\}^{k}}{k ! \Gamma\left(\frac{1-k}{2}\right)}=\frac{1}{\sqrt{\pi}} \exp \left\{-\frac{\left|\mathbf{r}_{b}-\mathbf{r}_{a}\right|^{2}}{4 i \hbar D_{2}\left(t_{b}-t_{a}\right)}\right\}
$$

which transforms Eq.(35) into

$$
\left.K_{L}^{(0)}\left(\mathbf{r}_{b} t_{b} \mid \mathbf{r}_{a} t_{a}\right)\right|_{\alpha=2}=\left(\frac{\hbar}{4 \pi i \hbar D_{2}\left(t_{b}-t_{a}\right)}\right)^{3 / 2} \exp \left\{-\frac{\left|\mathbf{r}_{b}-\mathbf{r}_{a}\right|^{2}}{4 i \hbar D_{2}\left(t_{b}-t_{a}\right)}\right\}
$$


Since $D_{2}=1 / 2 m$ we got the Feynman 3-D quantum mechanical kernel $K^{(0)}\left(\mathbf{r}_{b} t_{b} \mid \mathbf{r}_{a} t_{a}\right)$ of a particle with the mass $m$ (see, Problem 4-12, page 89 of Ref. [7]),

$\left.K^{(0)}\left(\mathbf{r}_{b} t_{b} \mid \mathbf{r}_{a} t_{a}\right) \equiv K_{L}^{(0)}\left(\mathbf{r}_{b} t_{b} \mid \mathbf{r}_{a} t_{a}\right)\right|_{\alpha=2}=\left(\frac{m}{2 \pi i \hbar\left(t_{b}-t_{a}\right)}\right)^{3 / 2} \exp \left\{\frac{i m\left|\mathbf{r}_{b}-\mathbf{r}_{a}\right|^{2}}{2 \hbar\left(t_{b}-t_{a}\right)}\right\}$.

Thus, the general equation (34) includes the Feynman 3-D kernel as a special case at $\alpha=2$.

\section{Transforms of a free particle kernel}

\subsection{The Laplace transform of a free particle kernel}

The Laplace in time transform $\widetilde{K}_{L}^{(0)}(x, s)$ of 1-D free particle kernel is defined as

$$
\widetilde{K}_{L}^{(0)}(x, s)=\int_{0}^{\infty} d \tau e^{-s \tau} K_{L}^{(0)}(x, \tau)
$$

where $K_{L}^{(0)}(x, \tau)$ is given by Eq.(25).

Using Eq.(38) and applying the series expansion for the function $H_{2,2}^{1,1}$ yield

$$
\begin{aligned}
\widetilde{K}_{L}^{(0)}(x, s) & =\frac{1}{\alpha x} \sum_{k=0}^{\infty} \frac{\Gamma\left(\frac{1+k}{\alpha}\right)}{\Gamma\left(\frac{1+k}{2}\right) \Gamma\left(\frac{1-k}{2}\right)} \frac{(-1)^{k}}{k !} \cdot\left(\frac{1}{\hbar}\left(\frac{\hbar}{i D_{\alpha}}\right)^{1 / \alpha}|x|\right)^{1+k} \int_{0}^{\infty} d \tau e^{-s \tau} \tau^{-\frac{1+k}{\alpha}}= \\
& \frac{1}{\alpha x s} \sum_{k=0}^{\infty} \frac{\Gamma\left(\frac{1+k}{\alpha}\right) \Gamma\left(1-\frac{1+k}{\alpha}\right)}{\Gamma\left(\frac{1+k}{2}\right) \Gamma\left(\frac{1-k}{2}\right)} \frac{(-1)^{k}}{k !} \cdot\left(\frac{1}{\hbar}\left(\frac{\hbar s}{i D_{\alpha}}\right)^{1 / \alpha}|x|\right)^{1+k},
\end{aligned}
$$

where we took into account that

$$
\int_{0}^{\infty} d \tau e^{-s \tau} \tau^{-\frac{1+k}{\alpha}}=s^{\frac{1+k}{\alpha}-1} \Gamma\left(1-\frac{1+k}{\alpha}\right) .
$$


Applying the definition of the Fox's function $H_{3,2}^{1,2}$ (see Eqs.(58)-(601), Appendix A) we can write finally the Laplace transform $\widetilde{K}_{L}^{(0)}(x, s)$ in terms of $H$-function

$$
\widetilde{K}_{L}^{(0)}(x, s)=\frac{1}{\alpha x s} H_{3,2}^{1,2}\left[\frac{1}{\hbar}\left(\frac{\hbar s}{i D_{\alpha}}\right)^{1 / \alpha}|x| \mid \begin{array}{c}
(1,1 / \alpha),(0,-1 / \alpha),(1,1 / 2) \\
(1,1),(1,1 / 2)
\end{array}\right] .
$$

Putting in Eq. (40) $\alpha=2$ and using the series expansion for the $H_{3,2}^{1,2}$ function we obtain the Laplace transform of a free particle kernel for the standard quantum mechanics

$$
\left.\widetilde{K}^{(0)}(x, s) \equiv \widetilde{K}_{L}^{(0)}(x, s)\right|_{\alpha=2}=\sqrt{\frac{m}{2 s i \hbar}} \exp \left\{-\sqrt{\frac{2 m s}{i \hbar}}|x|\right\} .
$$

\subsection{The energy-time transformation}

In quantum mechanics an important role plays the Fourier transform of the kernel in the time variable, which is the fixed-energy kernel $k_{L}\left(x_{2}, x_{1} ; E\right)$

$$
k_{L}\left(x_{2}, x_{1} ; E\right)=\int_{t_{1}}^{\infty} d t_{2} e^{(i / \hbar) E\left(t_{2}-t_{1}\right)} \cdot K_{L}\left(x_{2} t_{2} \mid x_{1} t_{1}\right),
$$

where $K_{L}\left(x_{2} t_{2} \mid x_{1} t_{1}\right)$ is given by Eq.(12).

To make the integral convergent, we have to move the energy into the upper complex half-plane by an infinitesimal amount $\epsilon$. Then the Lévy fixedenergy kernel becomes

$$
k_{L}\left(x_{2}, x_{1} ; E\right)=\sum_{n=1}^{\infty} \phi_{n}\left(x_{2}\right) \phi_{n}^{*}\left(x_{1}\right) \cdot \frac{i \hbar}{E-E_{n}+i \epsilon},
$$

here $\phi_{n}(x)$ and $E_{n}$ are the eigenfunctions and eigenenergies of the Hamiltonian $H_{\alpha}$ defined by Eq. (13).

The small $i \epsilon$-shift in the energy $E$ in (43) may be thought of as being attached to each of the energies $E_{n}$ which are thus placed by an infinitesimal piece below the real energy axis. When doing the Fourier integral (42) the exponential $e^{-(i / \hbar) E\left(t_{2}-t_{1}\right)}$ makes it always possible to close the integration contour along the energy axis by an infinite semicircle in the complex energy 
plane, which lies in the upper half-plane for $t_{2}<t_{1}$ and in the lower halfplane for $t_{2}>t_{1}$. The $i \epsilon$-shift guarantees that for $t_{2}<t_{1}$, there is no pole inside the closed contour making the kernel vanish. For $t_{2}>t_{1}$, on the other hand, poles in the lower half-plane give, via Cauchy's residue theorem, the spectral representation of the kernel (43).

We see that the fixed-energy kernel $k_{L}\left(x_{2}, x_{1} ; E\right)$ and the kernel $K_{L}\left(x_{2} t_{2} \mid x_{1} t_{1}\right)$ related each other by the inverse energy Fourier transform

$$
K_{L}\left(x_{2} t_{2} \mid x_{1} t_{1}\right)=\frac{1}{2 \pi \hbar} \int_{-\infty}^{\infty} d E e^{-(i / \hbar) E\left(t_{2}-t_{1}\right)} \cdot k_{L}\left(x_{2}, x_{1} ; E\right) .
$$

Let us calculate a free particle fixed-energy kernel $k_{L}^{(0)}\left(\mathbf{r}_{2}, \mathbf{r}_{1} ; E\right)$ defined as follows

$$
k_{L}^{(0)}\left(\mathbf{r}_{2}, \mathbf{r}_{1} ; E\right)=\int_{t_{1}}^{\infty} d t_{2} e^{(i / \hbar) E\left(t_{2}-t_{1}\right)} \cdot K_{L}^{(0)}\left(\mathbf{r}_{2} t_{2} \mid \mathbf{r}_{1} t_{1}\right) .
$$

With help of Eq.(34) we have

$$
\begin{gathered}
k_{L}^{(0)}\left(\mathbf{r}_{2}, \mathbf{r}_{1} ; E\right)=-\frac{1}{2 \pi \alpha} \frac{1}{\left|\mathbf{r}_{2}-\mathbf{r}_{1}\right|^{3}} \int_{t_{1}}^{\infty} d t_{2} e^{(i / \hbar) E\left(t_{2}-t_{1}\right)} \times \\
H_{3,3}^{1,2}\left[\frac{1}{\hbar}\left(\frac{\hbar}{i D_{\alpha}\left(t_{2}-t_{1}\right)}\right)^{1 / \alpha}\left|\mathbf{r}_{2}-\mathbf{r}_{1}\right| \mid \begin{array}{c}
(1,1),(1,1 / \alpha),(1,1 / 2) \\
(1,1),(1,1 / 2),(2,1)
\end{array}\right] .
\end{gathered}
$$

Using the Property 12.2.8 of $H$-function (see Appendix A) yields

$$
\begin{aligned}
& k_{L}^{(0)}\left(\mathbf{r}_{2}, \mathbf{r}_{1} ; E\right)=\frac{\hbar}{2 \pi \alpha i E\left|\mathbf{r}_{2}-\mathbf{r}_{1}\right|^{3}} \times \\
& H_{4,3}^{1,3}\left[\frac{1}{\hbar}\left(-\frac{E}{D_{\alpha}}\right)^{1 / \alpha}\left|\mathbf{r}_{2}-\mathbf{r}_{1}\right| \mid \begin{array}{c}
(0,-1 / \alpha),(1,1),(1,1 / \alpha),(1,1 / 2) \\
(1,1),(1,1 / 2),(2,1)
\end{array}\right] .
\end{aligned}
$$

Thus the expression for the fixed-energy kernel $k_{L}^{(0)}\left(\mathbf{r}_{2}, \mathbf{r}_{1} ; E\right)$ involves the $H_{4,3}^{1,3}$ Fox's $H$-function. The inverse energy Fourier transform defined by Eq.(44) and the expression (47) allow to obtain the following alternative representation for the quantum mechanical kernel $K_{L}^{(0)}\left(\mathbf{r}_{2} t_{2} \mid \mathbf{r}_{1} t_{1}\right)$ 


$$
\begin{gathered}
K_{L}^{(0)}\left(\mathbf{r}_{2} t_{2} \mid \mathbf{r}_{1} t_{1}\right)=\frac{\hbar}{(2 \pi)^{2} \alpha i\left|\mathbf{r}_{2}-\mathbf{r}_{1}\right|^{3}} \int_{-\infty}^{\infty} d E \frac{e^{-(i / \hbar) E\left(t_{2}-t_{1}\right)}}{E} \times \\
H_{4,3}^{1,3}\left[\frac{1}{\hbar}\left(-\frac{E}{D_{\alpha}}\right)^{1 / \alpha}\left|\mathbf{r}_{2}-\mathbf{r}_{1}\right| \mid \begin{array}{c}
(0,-1 / \alpha),(1,1),(1,1 / \alpha),(1,1 / 2)] \\
(1,1),(1,1 / 2),(2,1)
\end{array}\right] .
\end{gathered}
$$

If we put $\alpha=2$ Eq. (48) goes over into the standard quantum mechanical fixed-energy kernel $\left.k_{L}^{(0)}\left(\mathbf{r}_{2}, \mathbf{r}_{1} ; E\right)\right|_{\alpha=2}=k^{(0)}\left(\mathbf{r}_{2}, \mathbf{r}_{1} ; E\right)$. Indeed, setting in Eq.(48) $\alpha=2$ and taking into account that in accordance with the definition of $H$-function (see Eqs.(58)-(60), Appendix A)

$$
\begin{gathered}
H_{4,3}^{1,3}\left[\kappa \cdot\left|\mathbf{r}_{2}-\mathbf{r}_{1}\right| \mid \begin{array}{c}
(0,-1 / 2),(1,1),(1,1 / 2),(1,1 / 2) \\
(1,1),(1,1 / 2),(2,1)
\end{array}\right]= \\
-\left(\kappa \cdot\left|\mathbf{r}_{2}-\mathbf{r}_{1}\right|\right)^{2} \exp \left(-\kappa \cdot\left|\mathbf{r}_{2}-\mathbf{r}_{1}\right|\right)
\end{gathered}
$$

we find the well known equation for the fixed-energy kernel (see, for example Eq.(1.390) at the space dimension $D=3$, Chapter 1, [12])

$$
\begin{aligned}
k^{(0)}\left(\mathbf{r}_{2}, \mathbf{r}_{1} ; E\right)= & \frac{2 m}{4 \pi \hbar i \kappa^{2}\left|\mathbf{r}_{2}-\mathbf{r}_{1}\right|^{3}} \times\left(\kappa \cdot\left|\mathbf{r}_{2}-\mathbf{r}_{1}\right|\right)^{2} \exp \left(-\kappa \cdot\left|\mathbf{r}_{2}-\mathbf{r}_{1}\right|\right)= \\
& \frac{m}{2 \pi \hbar i\left|\mathbf{r}_{2}-\mathbf{r}_{1}\right|} \times \exp \left(-\kappa \cdot\left|\mathbf{r}_{2}-\mathbf{r}_{1}\right|\right),
\end{aligned}
$$

where for simplicity the notation $\kappa=\frac{1}{\hbar}\left(-\frac{E}{D_{\alpha}}\right)^{1 / \alpha}$ has been introduced.

\subsection{Momentum representation}

To find quantum kernel in the momentum representation let us introduce the momentum space wave function $\varphi(\mathbf{p}, t)$,

$$
\varphi(\mathbf{p}, t)=\int d \mathbf{r} e^{-\frac{i}{\hbar} \mathbf{p r}} \psi(\mathbf{r}, t)
$$

where $\psi(\mathbf{r}, t)$ is the wave function in coordinate representation 


$$
\psi(\mathbf{r}, t)=\frac{1}{(2 \pi \hbar)^{3}} \int d \mathbf{p} e^{\frac{i}{\hbar} \mathbf{p r}} \varphi(\mathbf{p}, t)
$$

Then we consider the 3-D generalization of Eq.(41)

$$
\psi\left(\mathbf{r}_{b}, t_{b}\right)=\int d \mathbf{r}_{a} K_{L}\left(\mathbf{r}_{b} t_{b} \mid \mathbf{r}_{a} t_{a}\right) \cdot \psi\left(\mathbf{r}_{a}, t_{a}\right) .
$$

Substituting the equation for the wave function in coordinate representation $\psi(\mathbf{r}, t)$ in terms of the wave function in the momentum representation $\varphi(\mathbf{p}, t)$ yields

$$
\varphi\left(\mathbf{p}_{b}, t_{b}\right)=\int d \mathbf{p}_{a} \mathcal{K}_{L}\left(\mathbf{p}_{b} t_{b} \mid \mathbf{p}_{a} t_{a}\right) \cdot \varphi\left(\mathbf{p}_{a}, t_{a}\right)
$$

where the kernel in the momentum representation $\mathcal{K}_{L}\left(\mathbf{p}_{b} t_{b} \mid \mathbf{p}_{a} t_{a}\right)$ is defined in terms of the kernel in coordinate representation $K_{L}\left(\mathbf{r}_{b} t_{b} \mid \mathbf{r}_{a} t_{a}\right)$ as follows

$$
\mathcal{K}_{L}\left(\mathbf{p}_{b} t_{b} \mid \mathbf{p}_{a} t_{a}\right)=\int d \mathbf{r}_{b} d \mathbf{r}_{a} e^{-\frac{i}{\hbar} \mathbf{p}_{b} \mathbf{r}_{b}+\frac{i}{\hbar} \mathbf{p}_{a} \mathbf{r}_{a}} \cdot K_{L}\left(\mathbf{r}_{b} t_{b} \mid \mathbf{r}_{a} t_{a}\right)
$$

For example, for a free particle we have

$$
\begin{aligned}
& \mathcal{K}_{L}^{(0)}\left(\mathbf{p}_{b} t_{b} \mid \mathbf{p}_{a} t_{a}\right)=\int d \mathbf{r}_{b} d \mathbf{r}_{a} e^{-\frac{i}{\hbar} \mathbf{p}_{b} \mathbf{r}_{b}+\frac{i}{\hbar} \mathbf{p}_{a} \mathbf{r}_{a}} \cdot K_{L}^{(0)}\left(\mathbf{r}_{b} t_{b} \mid \mathbf{r}_{a} t_{a}\right)= \\
= & (2 \pi \hbar)^{3} \delta\left(\mathbf{p}_{a}-\mathbf{p}_{b}\right) \cdot \exp \left\{-\frac{i}{\hbar} D_{\alpha}\left|\mathbf{p}_{a}\right|^{\alpha}\left(t_{b}-t_{a}\right)\right\}, \quad \text { for } \quad t_{b}>t_{a} .
\end{aligned}
$$

We see from Eq.(150) that a free particle kernel in the momentum space is expressed in terms of exponential function, while the kernel in the coordinate space has more complicated form.

\section{$5 \quad$ Particle in a box}

Now we are going to consider the impact of integration over the Lévy flights paths on quantum kernel for a particle in 1-D box of length $2 a$ confined by infinitely high walls at $x=-a$ and $x=a$. From the eigenvalues [10]

$$
E_{n}=D_{\alpha}\left(\frac{\pi \hbar}{a}\right)^{\alpha} n^{\alpha}, \quad 1<\alpha \leq 2,
$$


with the principal quantum number $n=1,2,3 \ldots$, and corresponding eigenfunctions [10]

$$
\psi_{n}(x)=\frac{1}{\sqrt{a}} \sin \frac{n \pi x}{a}
$$

it follows that the quantum kernel for a particle in the box has the form

$$
K_{\text {box }}\left(x_{b} t \mid x_{a} 0\right)=\frac{1}{a} \sum_{n=1}^{\infty} \sin \frac{n \pi x_{b}}{a} \sin \frac{n \pi x_{a}}{a} \exp \left\{-\frac{i}{\hbar} D_{\alpha}\left(\frac{\pi \hbar}{a}\right)^{\alpha} n^{\alpha} t\right\} .
$$

Here $x_{a}$ and $x_{b}$ are initial and final particle positions in the box. Note that the eigenfunctions $\psi_{n}(x)$ given by Eq.(52) guarantee that the kernel satisfies the boundary conditions

$$
K_{\text {box }}\left(x_{b}=a, t \mid x_{a}, 0\right)=K_{\text {box }}\left(x_{b}, t \mid x_{a}=-a, 0\right)=0,
$$

enforced by the two infinite walls at $x=-a$ and $x=a$ at all times.

Then Eq.(53) can be expressed as

$$
\begin{gathered}
K_{\text {box }}\left(x_{b} t \mid x_{a} 0\right)= \\
\frac{1}{2 a} \sum_{n=1}^{\infty}\left\{\cos \frac{n \pi}{a}\left(x_{b}-x_{a}\right)-\cos \frac{n \pi}{a}\left(x_{b}+x_{a}\right)\right\} \exp \left\{-\frac{i}{\hbar} D_{\alpha}\left(\frac{\pi \hbar}{a}\right)^{\alpha} n^{\alpha} t\right\}
\end{gathered}
$$

The next steps to transform the above equation are

$$
\begin{gathered}
K_{\text {box }}\left(x_{b} t \mid x_{a} 0\right)= \\
\frac{\pi}{2 \pi \hbar a} \sum_{l=-\infty}^{\infty} \int_{-\infty}^{\infty} d p \delta\left(\frac{p}{\hbar}-\frac{\pi}{a} l\right)\left\{\exp \left[\frac{i p\left(x_{b}-x_{a}\right)}{\hbar}\right]-\exp \left[\frac{-i p\left(x_{b}+x_{a}\right)}{\hbar}\right]\right\} \times \\
\exp \left\{-\frac{i}{\hbar} D_{\alpha}|p|^{\alpha} t\right\}= \\
\frac{1}{2 \pi \hbar} \sum_{l=-\infty}^{\infty} \int_{-\infty}^{\infty} d p\left\{\exp \left[\frac{i p\left(x_{b}-x_{a}+2 l a\right)}{\hbar}\right]-\exp \left[\frac{-i p\left(x_{b}+x_{a}-2 l a\right)}{\hbar}\right]\right\} \times
\end{gathered}
$$




$$
\exp \left\{-\frac{i}{\hbar} D_{\alpha}|p|^{\alpha} t\right\}
$$

where the Poisson summation formula ${ }^{3}$ has been applied.

If we take into account the definition of a free particle kernel $K_{L}^{(0)}\left(x_{b} t \mid x_{a} 0\right)$ given by Eq.(10) the kernel for the particle in the box $K_{\text {box }}\left(x_{b} t \mid x_{a} 0\right)$ becomes

$$
K_{\text {box }}\left(x_{b} t \mid x_{a} 0\right)=\sum_{l=-\infty}^{\infty}\left\{K_{L}^{(0)}\left(x_{b}+2 l a, t \mid x_{a} 0\right)-K_{L}^{(0)}\left(-x_{b}+2 l a, t \mid x_{a} 0\right)\right\} .
$$

In terms of Fox's $H$-function $K_{\text {box }}\left(x_{b} t \mid x_{a} 0\right)$ is

$$
\begin{gathered}
K_{\text {box }}\left(x_{b} t \mid x_{a} 0\right)=\frac{1}{\alpha}\left(\frac{\hbar}{\left(\hbar / i D_{\alpha} t\right)^{1 / \alpha}}\right)^{-1} \times \\
\sum_{l=-\infty}^{\infty}\left\{H_{2,2}^{1,1}\left[\frac{1}{\hbar}\left(\frac{\hbar}{i D_{\alpha} t}\right)^{1 / \alpha}\left|x_{b}-x_{a}+2 l a\right| \mid \begin{array}{c}
(1-1 / \alpha, 1 / \alpha),(1 / 2,1 / 2) \\
(0,1),(1 / 2,1 / 2)
\end{array}\right]-\right. \\
\left.H_{2,2}^{1,1}\left[\frac{1}{\hbar}\left(\frac{\hbar}{i D_{\alpha} t}\right)^{1 / \alpha}\left|-x_{b}-x_{a}+2 l a\right| \mid \begin{array}{c}
(1-1 / \alpha, 1 / \alpha),(1 / 2,1 / 2) \\
(0,1),(1 / 2,1 / 2)
\end{array}\right]\right\} .
\end{gathered}
$$

To understand Eq. (57) for $K_{\text {box }}\left(x_{b} t \mid x_{a} 0\right)$ from point of view of physics let us remind the analogy with the method of image charges in electrostatics. Following the method of image charges one can account the conducted plate by putting a negative charge complementing the positive charge. In this case, the image method yields an infinite number of charges of alternating signs. The original positive charge gives rise to two negative charges which are each an image corresponding to one of the two conducted plates. These images generate mirror images corresponding to other conducted plate and the image generation process has going on up to infinity. The quantum problem of particle in the box can be treated similar to the electrostatics problem of a charge between two parallel conducted plates. To apply electrostatic analogy

$$
\frac{\pi}{a} \sum_{l=-\infty}^{\infty} \delta\left(\frac{p}{\hbar}-\frac{\pi}{a} l\right)=\sum_{l=-\infty}^{\infty} \exp \left\{i \frac{2 p a}{\hbar} l\right\}
$$

where $\delta$ is the Dirac delta function. 
to the quantum kernel let us start with simple physical situation of just one infinite wall and take a look at all paths running between $x_{a}$ and $x_{b}$ in time $t$. As can be seen from the space-time diagram in Fig.1 there is quantum path which does not cross the wall and which therefore contributes to the path integral. However between $x_{a}$ and $x_{b}$ there exist also path which crosses the wall an even number of times. Since this path goes through the forbidden region, it should not contribute to the path integral. To eliminate contribution of those paths we have to apply a procedure to ensure that only quantum paths not crossing the wall will be taken into account. The procedure can be realized as follows. At first we write down a free particle kernel $K_{L}^{(0)}\left(x_{b} t \mid x_{a} 0\right)$ which disregards the wall. Then we have to subtract from $K_{L}^{(0)}\left(x_{b} t \mid x_{a} 0\right)$ the contribution of all paths which cross the wall. This can be done by constructing a free particle path integral with the same classical mechanical action as one which contributes to the $K_{L}^{(0)}\left(x_{b} t \mid x_{a} 0\right)$. We take the original path up to the last crossing with the wall and then to continue along the mirror image of the original path. We thus end up at the mirror image $-x_{b}$ of the original end point $x_{b}$. Note that a path running from $x_{a}$ to $-x_{b}$ necessarily crosses the wall at least once. Therefore substracting a free particle kernel between $x_{a}$ and $-x_{b}$ eliminates contribution from all paths which do not remain in the physical region $x>0$. Finally we conclude that the kernel $K_{\text {wall }}\left(x_{b} t \mid x_{a} 0\right)$ in presence of a wall can be expressed by substracting a free particle kernel going from $x_{a}$ to the mirror image $-x_{b}$ from a free particle kernel going from $x_{a}$ to $x_{b}$, see Fig.1,

$$
K_{\text {wall }}\left(x_{b} t \mid x_{a} 0\right)=K_{L}^{(0)}\left(x_{b} t \mid x_{a} 0\right)-K_{L}^{(0)}\left(-x_{b} t \mid x_{a} 0\right) .
$$

Expressing the kernel of a particle in the box in terms of free kernel works exactly in the same way. Indeed, a path intersecting both walls is subtracted twice, i.e. one time too often. Therefore, one contribution has to be restored which is done by adding another end point. Continuing the procedure one ends up with an infinite number of end points. The general rule to attribute a sign to each end point is that each reflection at a wall leads to factor -1 . This procedure immediately gives us Eq.(57).

Using the way of consideration which leads from Eq.(17) to Eq.(30) we can see that at $\alpha=2$ the Lévy quantum kernel Eq.(157) goed over into the well known Feynman kernel for a particle in a box (see, for example Eq.(6.19) and (6.20) in the [12]). 


\section{Conclusion}

We have studied an impact of integration over the paths of the Lévy flights on the structure of a free particle quantum mechanical kernel. Analytical expression of a free particle 1-D quantum kernel has been obtained in term of the Fox $H$-function. The 3-D generalization has been presented as well. The Laplace, energy-time and momentum transforms of a free particle kernel have been obtained and discussed. We have found the quantum kernel for a particle in the box.

While the Feynman path integral is in fact integration over the Brownianlike paths the Lévy path integral is the integral over the Lévy flights trajectories. The path integral over the Lévy flights generalizes at $\alpha<2$ and becomes at $\alpha=2$ the Feynman path integral. The new equations (26), (34), (40), (48), (501) and (57) at the special case $\alpha=2$ go over into the well known quantum mechanical equations for a free particle kernel and a particle in the box kernel.

\section{Appendix A}

\subsection{Fox $H$-function}

In Sec.3 we have expressed a free particle kernel in the term of Fox function $H_{2,2}^{1,1}$. Apart from the quiet natural way in which the Fox $H$-function enters in the path integrals over the Lévy flights, its fractional derivatives and integrals are easily calculated by formally manipulating the parameters in the $H$ function. That is why the Fox's $H$-function is important and useful for the Lévy path integral calculations.

Some properties of the $H$-function in connection with Mellin-Barnes integrals were investigated by Barnes [17, Mellin [18, Dixon and Ferrar [19]. In an attempt to unify and extend the existing results on symmetrical Fourier kernels, Fox [13] has defined the $H$-function in terms of a general MellinBarnes type integral. Asymptotic expansions and analytic continuations of the Fox function and its special cases were derived by Braaksma [20]. Many properties of the $H$-function are reported in the book [14 along with applications to statistics.

Fox's $H$-function is defined by the Mellin-Barnes type integral [13, 20] (we follow the notations of the book [14]) 


$$
\begin{gathered}
H_{p, q}^{m, n}(z)=H_{p, q}^{m, n}\left[\begin{array}{c}
\left(a_{p}, A_{p}\right) \\
\left(b_{q}, B_{q}\right)
\end{array}\right]= \\
H_{p, q}^{m, n}\left[z \mid \begin{array}{c}
\left(a_{1}, A_{1}\right), \ldots,\left(a_{p}, A_{p}\right) \\
\left(b_{1}, B_{1}\right), \ldots,\left(b_{q}, B_{q}\right)
\end{array}\right]=\frac{1}{2 \pi i} \int_{L} d s z^{s} \chi(s),
\end{gathered}
$$

where function $\chi(s)$ is given by

$$
\chi(s)=\frac{\prod_{j=1}^{m} \Gamma\left(b_{j}-B_{j} s\right) \prod_{j=1}^{n} \Gamma\left(1-a_{j}+A_{j} s\right)}{\prod_{j=m+1}^{q} \Gamma\left(1-b_{j}+B_{j} s\right) \prod_{j=n+1}^{p} \Gamma\left(a_{j}-A_{j} s\right)},
$$

and

$$
z^{s}=\exp \{s \log |z|+i \arg z\}
$$

here $m, n p$ and $q$ are non negative integers satisfying $0 \leq n \leq p, 1 \leq m \leq q$; and the empty products are interpreted as unity. The parameters $A_{j}(j=$ $1, \ldots, p)$ and $B_{j}(j=1, \ldots, q)$ are positive numbers; $a_{j}(j=1, \ldots, p)$ and $b_{j}$ $(j=1, \ldots, p)$ are complex numbers such that

$$
A_{j}\left(b_{h}+\nu\right) \neq B_{h}\left(a_{j}-\lambda-1\right),
$$

for $\nu, \lambda=0,1, \ldots ; h=1, \ldots, m ; j=1, \ldots, n$.

The $L$ is a contour separating the points

$$
s=\left(\frac{b_{j}+\nu}{B_{j}}\right), \quad(j=1, \ldots, m ; \quad \nu=0,1, \ldots),
$$

which are the poles of $\Gamma\left(b_{j}-B_{j} s\right)(j=1, \ldots, m)$, from the points

$$
s=\left(\frac{a_{j}-\nu-1}{A_{j}}\right), \quad(j=1, \ldots, n ; \quad \nu=0,1, \ldots)
$$

which are the poles of $\Gamma\left(1-a_{j}+A_{j} s\right)(j=1, \ldots, n)$. The contor $L$ exists on account of (60). These assumptions will be retained throughout.

In the contracted form the $H$ function in Eq.(58) will be denoted by one of the following notations: 


$$
H(z), \quad H_{p, q}^{m, n}(z), \quad H_{p, q}^{m, n}\left[\begin{array}{c}
\left(a_{p}, A_{p}\right) \\
\left(b_{q}, B_{q}\right)
\end{array}\right] .
$$

The Fox $H$-function is an analytic function of $z$ which makes sense (i) for every $z \neq 0$ if $\mu>0$ and (ii) for $0<|z|<\beta^{-1}$ if $\mu=0$, where

$$
\mu=\sum_{j=1}^{q} B_{j}-\sum_{j=1}^{p} A_{j}
$$

and

$$
\beta=\prod_{j=1}^{p} A_{j}^{A_{j}} \prod_{j=1}^{q} B_{j}^{-B_{j}} .
$$

Due to the occurrence of the factor $z^{s}$ in Eq.(58), the $H$-function is in general multiple-valued, but is one-valued on the Riemann surface of $\log z$.

The $H$ function is a generalization of Meijer's $G$ function [21], which is also defined by a Mellin-Barnes integral. The $H$ function reduces to the $G$ function if $A_{j}=1$ and $B_{k}=1$ for all $j=1,2, \ldots, p$ and $k=1,2, \ldots, q$,

$$
G_{p, q}^{m, n}(z)=H_{p, q}^{m, n}\left[z \mid \begin{array}{c}
\left(a_{1}, 1\right), \ldots,\left(a_{p}, 1\right) \\
\left(b_{1}, 1\right), \ldots,\left(b_{q}, 1\right)
\end{array}\right] .
$$

If further $m=1$ and $p \leq q$, then the $H$ function is expressible by

$$
\begin{aligned}
H_{p, q}^{1, n}\left[z \mid \begin{array}{c}
\left(a_{1}, 1\right) \ldots\left(a_{p}, 1\right) \\
\left(b_{1}, 1\right) \ldots\left(b_{q}, 1\right)
\end{array}\right]=\frac{\prod_{j=1}^{n} \Gamma\left(1+b_{1}-a_{j}\right) z^{b_{1}}}{\prod_{j=2}^{q} \Gamma\left(1+b_{1}-b_{j}\right) \prod_{j=n+1}^{p} \Gamma\left(a_{j}-b_{1}\right)} \times \\
\quad \times{ }_{p} F_{q-1}\left(\begin{array}{l}
1+b_{1}-a_{1}, \ldots, 1+b_{1}-a_{p} \\
1+b_{1}-b_{2}, \ldots, 1+b_{1}-b_{q}
\end{array} ;(-1)^{p-n-1} z\right),
\end{aligned}
$$

in terms of generalized hypergeometric functions ${ }_{p} F_{q}[14$. As far as many well-known special functions, such as error function, Bessel functions, Whittaker functions, Jacobi polynomials, and elliptic functions are included in the class of generalized hypergeometric functions, all of them can be expressed in the term of the Fox's $H$ function. 
To represent of $H$-unction in computable form let us consider the case when the poles $s=\left(b_{j}+\nu\right) / B_{j}(j=1, \ldots, m ; \nu=0,1, \ldots)$ of $\prod_{j=1}^{m}{ }^{\prime} \Gamma\left(b_{j}-B_{j} s\right)$ are simple, that is, where

$$
\begin{aligned}
& B_{h}\left(b_{j}+\lambda\right) \neq B_{j}\left(b_{h}+\nu\right), \quad j \neq h, \\
& h=1, \ldots, m ; \quad \nu, \lambda=0,1,2, \ldots,
\end{aligned}
$$

and the prime means the product without the factor $j=h$. Then we obtain the following expansion for the $H$-function

$$
\begin{gathered}
H_{p, q}^{m, n}(z)=\sum_{h=1}^{m} \sum_{k=0}^{\infty} \frac{\prod_{j=1}^{n} \Gamma\left(1-a_{j}+A_{j} s_{h k}\right) \prod_{j=1}^{m}{ }^{\prime} \Gamma\left(b_{j}-B_{j} s_{h k}\right)}{\prod_{j=m+1}^{q} \Gamma\left(1-b_{j}+B_{j} s_{h k}\right) \prod_{j=n+1}^{p} \Gamma\left(a_{j}-A_{j} s_{h k}\right)} \frac{(-1)^{k}}{k !} \frac{z^{s_{h k}}}{B_{h}} \\
s_{h k}=\left(b_{h}+k\right) / B_{h}
\end{gathered}
$$

which exists for all $z \neq 0$ if $\mu>0$ and for $0<|z|, \beta^{-1}$ if $\mu=0$, where $\mu$ and $\beta$ are given by Eqs.(61) and (62).

The formula (63) can be used to calculate the special values of the $H$ function and to derive the asymptotic behavior for $z \rightarrow 0$.

\subsection{Some identities of the $H$-function}

The $H$-function possesses many interesting properties that are helpful for the calculations with the path integrals over Lévy flights. We present the list of mainly used properties of the $H$-function. The results of this section follow readily from the definition of the $H$-function given by Eq.(58) and hence no proofs are given here.

Property 12.2.1 The $H$-function is symmetric in the pairs $\left(a_{1}, A_{1}\right), \ldots,\left(a_{n}, A_{n}\right)$, likewise $\left(a_{n+1}, A_{n+1}\right), \ldots,\left(a_{p}, A_{p}\right)$; in $\left(b_{1}, B_{1}\right), \ldots,\left(b_{n}, B_{n}\right)$ and in $\left(b_{m+1}, B_{m+1}\right), \ldots,\left(b_{q}, B_{q}\right)$.

Property 12.2.2 If one of the $\left(a_{j}, A_{j}\right)(j=1, \ldots, n)$ is equal to one of the $\left(b_{j}, B_{j}\right)(j=m+1, \ldots, q)$ or one of the $\left(b_{j}, B_{j}\right)(j=1, \ldots, m)$ is equal to one of the $\left.\left(a_{j}, A_{j}\right)(j=n+1, \ldots, p)\right]$, then the $H$-function reduces to one of the lower order, and $p, q$ and $n$ (or) $m$ decrease by unity. 
Thus, we have the following reduction formula:

$$
\begin{gathered}
H_{p, q}^{m, n}\left[\begin{array}{cc}
z \mid & \left(a_{1}, A_{1}\right), \ldots,\left(a_{p}, A_{p}\right) \\
& \left(b_{1}, B_{1}\right), \ldots,\left(b_{q-1}, B_{q-1}\right),\left(a_{1}, A_{1}\right)
\end{array}\right]= \\
H_{p-1, q-1}^{m, n-1}\left[\begin{array}{cc}
z \mid & \left(a_{2}, A_{2}\right), \ldots,\left(a_{p}, A_{p}\right) \\
& \left(b_{1}, B_{1}\right), \ldots,\left(b_{q-1}, B_{q-1}\right)
\end{array}\right],
\end{gathered}
$$

provided $n \geq 1$ and $q>m$.

Property 12.2.3

$$
\begin{gathered}
H_{p, q}^{m, n}\left(z \mid \begin{array}{c}
\left(a_{1}, A_{1}\right), \ldots,\left(a_{p}, A_{p}\right) \\
\left(b_{1}, B_{1}\right), \ldots,\left(b_{q}, B_{q}\right)
\end{array}\right)= \\
H_{q, p}^{n, m}\left(\frac{1}{z} \mid \begin{array}{c}
\left(1-b_{1}, B_{1}\right), \ldots,\left(1-b_{q}, B_{q}\right) \\
\left(1-a_{1}, A_{1}\right), \ldots,\left(1-a_{p}, A_{p}\right)
\end{array}\right),
\end{gathered}
$$

This is an important property of the $H$-function because it enables us to transform an $H$-function with $\mu=\sum_{j=1}^{m} B_{j}-\sum_{j=1}^{n} A_{j}>0$ and $\arg x$ to one with $\mu<0$ and $\arg (1 / x)$ and vice versa.

Property 12.2.4

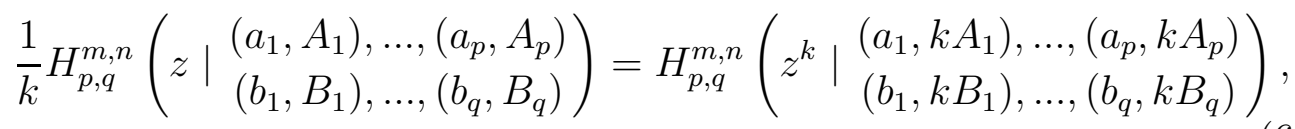

where $k>0$.

Property 12.2.5

$$
\begin{gathered}
z^{\sigma} H_{p, q}^{m, n}\left(z \mid \begin{array}{c}
\left(a_{1}, A_{1}\right), \ldots,\left(a_{p}, A_{p}\right) \\
\left(b_{1}, B_{1}\right), \ldots,\left(b_{q}, B_{q}\right)
\end{array}\right)= \\
H_{p, q}^{m, n}\left(z \mid \begin{array}{c}
\left(a_{1}+\sigma A_{1}, A_{1}\right), \ldots,\left(a_{p}+\sigma A_{p}, A_{p}\right) \\
\left(b_{1}+\sigma B_{1}, B_{1}\right), \ldots,\left(b_{q}+\sigma B_{q}, B_{q}\right)
\end{array}\right),
\end{gathered}
$$

Property 12.2.6 


$$
H_{p+1, q+1}^{m, n+1}\left(z \mid \begin{array}{c}
(0, \gamma), \ldots,\left(a_{p}, A_{p}\right) \\
\left(b_{q}, B_{q}\right), \ldots,(r, \gamma)
\end{array}\right)=(-1)^{r} H_{p+1, q+1}^{m+1, n}\left(z \mid \begin{array}{c}
\left(a_{p}, A_{p}\right), \ldots,(0, \gamma) \\
(r, \gamma), \ldots,\left(b_{q}, B_{q}\right)
\end{array}\right)
$$

where $p \leq q$.

\section{Property 12.2.7}

$$
\begin{aligned}
& H_{p+1, q+1}^{m+1, n}\left(\begin{array}{c}
\left(a_{p}, A_{p}\right), \ldots,(1-r, \gamma) \\
(1, \gamma), \ldots,\left(b_{q}, B_{q}\right)
\end{array}\right)=
\end{aligned}
$$

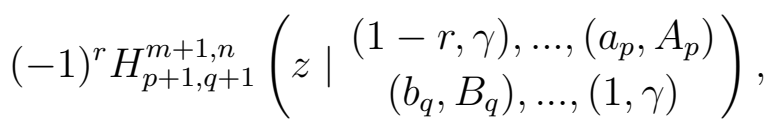

where $p \leq q$.

In the above Properties (12.1.2) - (12.1.6) the branches of the $H$-function are suitably chosen.

Property 12.2.8

$$
\begin{gathered}
\int d x x^{\alpha-1} e^{-\sigma x} H_{p, q}^{m, n}\left(\omega x^{r} \mid \begin{array}{c}
\left(a_{1}, A_{1}\right), \ldots,\left(a_{p}, A_{p}\right) \\
\left(b_{1}, B_{1}\right), \ldots,\left(b_{q}, B_{q}\right)
\end{array}\right)= \\
\sigma^{-\alpha} H_{p+1, q}^{m, n+1}\left(\frac{\omega}{\sigma^{r}} \mid \begin{array}{c}
(1-\alpha, r),\left(a_{1}, A_{1}\right), \ldots,\left(a_{p}, A_{p}\right) \\
\left(b_{1}, B_{1}\right), \ldots,\left(b_{q}, B_{q}\right)
\end{array}\right),
\end{gathered}
$$

where $m \cdot n \neq 0 ; r, p \geq 0 ; a, a^{*}>0 ;|\arg \omega|<a^{*} \pi / 2 ; \operatorname{Re} \alpha+r, \min _{1 \leq j \leq m}$ $\operatorname{Re}\left(b_{j} / B_{j}\right)>0$.

Property 12.2.9

$$
\begin{gathered}
{ }_{0} D_{z}^{\nu}\left[z^{\alpha} H_{p, q}^{m, n}\left((a z)^{\beta} \mid \begin{array}{c}
\left(a_{j}, A_{j}\right) \\
\left(b_{j}, B_{j}\right)
\end{array}\right)\right]= \\
=z^{\alpha-\nu} H_{p+1, q+1}^{m, n+1}\left((a z)^{\beta} \mid \begin{array}{c}
(-\alpha, \beta),\left(a_{j}, A_{j}\right) \\
\left(b_{j}, B_{j}\right),(\nu-\alpha, \beta)
\end{array}\right),
\end{gathered}
$$

where $\nu$ is arbitrary, $a, b>0$ and $\alpha+\beta \min \left(b_{j} / B_{j}\right)>-1(1 \leq j \leq m)$. Here the ${ }_{0} D_{z}^{\nu}$ notes fractional derivative of order $\nu$ (see for definition Refs. 22][25]). 


\section{References}

[1] G.M. Zaslavsky, Phys. Rep. 371, 461-580 (2002).

[2] R. Metzler, J. Klafter, Phys. Rep. 339, 1-77 (2000).

[3] F. Bardou, J-P Bouchaud, A. Aspect, C.Cohen-Tannoudji, Lévy Statistics and Laser Cooling, (Cambridge University Press, Cambridge, 2002).

[4] S. Schlipf, H. Katori, L. Perotti, H. Waiyher, Optics Express, 3, 97-103 (1998).

[5] G. Zimbardo, P. Veltri, G. Basile and S. Principato, Phys. Plasmas 2 (7), 2653 (1995).

[6] B.G. Klappauf, W.H. Oskay, D.A. Steck, M.G. Raizen, Phys. Rev. Lett. 81, 4044 (1998).

[7] R. P. Feynman and A.R. Hibbs, Quantum Mechanics and Path Integrals (McGraw-Hill, New York, 1965).

[8] R.P. Feynman, Statistical Mechanics, (Benjamin. Reading, Mass., 1972).

[9] N. Laskin, Phys. Rev. E 62, 3135 (2000).

[10] N. Laskin, Chaos 10, 780 (2000).

[11] N. Laskin, Phys. Rev. E 66, 056108 (2002).

[12] H. Kleinert, Path Integrals in Quantum Mechanics, Statistics and Polymer Physics, (World Scientific, Singapore 1990).

[13] C. Fox, Trans. Am. Math. Soc. 98, 395 (1961).

[14] A.M. Mathai and R.K. Saxena, The H-function with Applications in Statistics and Other Disciplines (Wiley Eastern, New Delhi, 1978).

[15] H.M. Srivastava, K. C. Gupta, and S.P. Goyal, The H-fuction of One and Two Variables with Applications (South Asian Publishers, New Delhi Madras, 1982).

[16] B.J. West and V. Seshadri, Physica, 113A, 203, (1982). 
[17] E.W. Barnes, Proc. Lond. Math. Soc. 6, 141 (1908).

[18] H.J. Mellin, Math. Ann., 68, 395 (1910).

[19] A.L. Dixon and W.L. Ferrat, Q.J. Math. Oxford Ser., 7, 81 (1936).

[20] B.L.J. Braaksma, Compos. Math., 15, 239 (1964).

[21] C.S. Meijer, Proc. Nedel. Akad. Wetensch, 49, 227, 344, 457, 632, 765, 936, 1062, 1165 (1).

[22] K.B. Oldham and J. Spanier, The Fractional Calculus (Academic Press, New York, 1974).

[23] K. Nishimoto, Fractional Calculus ( University of New Haven Press, New Haven CT, 1989).

[24] K.S. Miller and B. Ross, An Introduction to the Fractional Calculus and Fractional Differential Equations, (Wiley, New York, 1993).

[25] S.G. Samko, A.A. Kilbas and O.I. Marichev, Fractional Integrals and Derivatives, Theory and Applications, (Gordon and Breach, Amsterdam, 1993).

[26] G-L. Ingold, arXiv: quant-ph/020826.

\section{Figure caption}

Fig.1. A path crossing the wall is cancelled by a path running to the mirror point of the end point [26]. 